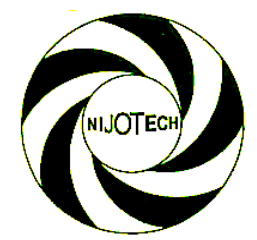

Nigerian Journal of Technology (NIJOTECH)

Vol. 38, No. 3, July 2019, pp. 732 - 743

Copyright@ Faculty of Engineering, University of Nigeria, Nsukka,

Print ISSN: 0331-8443, Electronic ISSN: 2467-8821

WwW.nijotech.com

http://dx.doi.org/10.4314/njt.v38i3.27

\title{
INVESTIGATING THE STEADY STATE STABILITY OF THE NIGERIAN 48- BUS SYSTEMS USING FACTS DEVICES
}

\author{
I. E. Nkan ${ }^{1, *}$, O. I. Okoro ${ }^{2}$, C. C. Awah ${ }^{3}$ and U. B. Akuru ${ }^{4}$ \\ 1, Dept. of Electrical/Electronic Engr'G, AKWa Ibom State Univ., Mpat-Enin, AKWA IBom STate, Nigeria

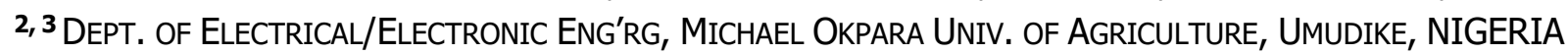 \\ 4, EMLAB (ElECTRICAL MACHINES LAB) | E260 DePARTEMENT OF ElECTRICAL AND ElECTRONIC ENGineERING, \\ STELLENBOSCH UNIVERSITY, STELLENBOSCH, 7600, SOUTH AFRICA \\ E-mail addresses: 1 imoenkan@yahoo.com, 2 oiokoro@yahoo.co.uk, 3 ccawah@ieee.org, \\ 4 18375243@sun.ac.za
}

\begin{abstract}
With the ongoing expansions and growth of the electric utility industry, including deregulation in Nigeria, numerous changes characterized by additional generating stations, increase in transmission lines and loads are experienced thereby pushing the transmission systems closer to their stability and thermal limits and hence, causing the transfer of reactive power during steady state operating conditions to constitute a major problem of voltage instability. Flexible ac transmission systems (FACTS) controllers have been utilized for finding solutions to various power system stability control issues such as voltage instability. This paper uses Static Synchronous Series Compensator (SSSC) and Thyristor Controlled Series Compensator (TCSC) to investigate the voltage magnitude profile, active and reactive power losses of the Nigerian 48-bus power system network for steady state stability enhancement using power system analysis toolbox (PSAT) in MATLAB environment. Optimal location of the FACTS devices was achieved through the computation of the voltage stability sensitivity factors (VSSF) for all the buses after continuation power flow (CPF) was carried out. Simulation results obtained without and with the FACTS devices revealed that the two FACTS devices especially TCSC has the capability to raise voltage profile of the buses and mitigate against power losses.
\end{abstract}

Keywords: CPF, FACTS, MATLAB, Optimal location, PSAT, SSSC, stability, TCSC, VSSF

\section{INTRODUCTION}

Power system stability is the ability of an electric power system, for a given initial operating condition, to regain a state of operating equilibrium after being subjected to a physical disturbance [1]. In voltage stability, this entails the ability of a power system to maintain an acceptable voltage profile throughout all the buses in the power system under normal condition and after experiencing a perturbation in the power network. A system enters a condition of voltage instability when a disturbance causes an increase in load demand or change in the system condition causes progressive and uncontrollable decline in voltage [2]. Now, more than ever, advanced technologies paramount for the reliable and secure operations of power systems [3] based on power electronics equipment called FACTS (Flexible AC Transmission Systems), provide proven technical solutions to address the operating challenges of power system instability being presented today. FACTS technologies allow for improved transmission system operation compared to the construction of new transmission lines. In [4], voltage collapse proximity index was computated for optimal placement of Thyristor Controlled Series Compensator (TCSC) and Static Synchronous Compensator (STATCOM) modeled in the IEEE 14 bus system using Power System Analysis Toolbox (PSAT) in MATLAB for improvement in the voltage stability of the system. In [5] also, reactive power loss sensitivity factor method was employed to

* Corresponding author, tel: +234-803-403-6949 
find out the best possible location of TCSC and Static Var Compensator (SVC) using power world simulator 8.0 on modified IEEE 9 bus system. Application of SVC using Owerri Transmission Company as case study for enhancement of power system voltage stability with the aid of reactive/capacitive power switching mechanism was presented in [6]. In [7], 28 bus, 330 $\mathrm{kV}$ Nigerian transmission system was modeled for the comparison of the voltage enhancement and loss reduction capabilities of STATCOM and Static Synchronous Series Compensator (SSSC) FACTS Controllers using MATLAB based programs. An overview of placement of TCSC for enhancement of power system stability was presented in [8]. Here, differential evolution technique was employed for the minimization of transmission losses and enhancement of stability of the power system. In [9], a systematic modular approach to incorporate series and shunt FACTS controllers in differential algebraic equation (DAE) model of multimachine power system in coordinated control manner for voltage stability analysis using MATLAB toolbox was presented. [10] reviewed the series and shunt compensation techniques in their discussion of the Nigerian $330 \mathrm{kV}$ transmission system associated with various small signal perturbations in Nigeria power system. In [11], STATCOM was optimally placed in the Nigerian $330 \mathrm{kV}$, 30-bus system using ant colony optimization metaheuristic technique for stability in the voltage profile of the buses. In [12], Unified Power Flow Controller (UPFC) was applied to Nigeria's $330 \mathrm{kV}, 28$-bus transmission system using MATLAB to minimize prolonged and frequent voltage instability in the network and enhance stability while in [13], Newton Raphson power flow iteration method using MATLAB was adopted to optimally placed SSSC in the Nigerian 28-bus system to control voltage magnitude at low voltage buses. In this paper, PSAT software will be used to model a larger Nigerian power system of 48 buses [18], 16 PV generators for load flow studies with 79 transmission lines which reflect the true nature of the ever expanding power system in Nigeria. Simulation studies will be carried out with the application of TCSC and SSSC to investigate the steady state stability of the system for improved system performance.

\section{MODELING OF TCSC AND SSSC}

\subsection{TCSC}

The functional model of TCSC is represented in Figure 1 with the terminals of the controller at $T_{K}$ and $T_{M}$. The fundamental frequency operation can be represented by the following set of equations, which includes the control system equations and sinusoidal currents in the controller.

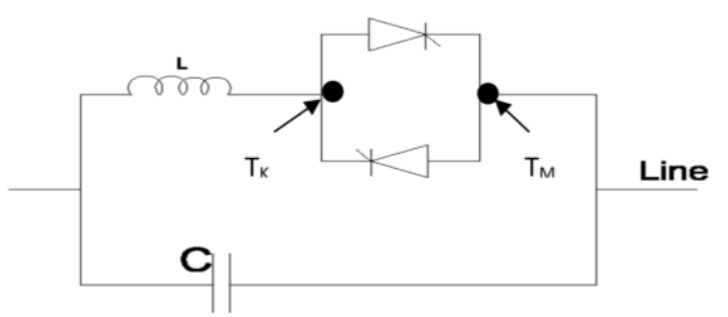

Figure 1: Thyristor Controlled Series Capacitor [14]

$$
\begin{array}{r}
{\left[x_{c}^{\prime}, \propto^{\prime}\right]^{T}=f\left(x_{c}, \propto, I, I_{\text {ref }}\right)} \\
P+V_{k} V_{m} B_{e} \sin \left(\delta_{k}-\delta_{m}\right)=0 \\
-V_{k}^{2} B_{e}+V_{k} V_{m} B_{e} \cos \left(\delta_{k}-\delta_{m}\right)-Q_{k}=0 \\
-V_{m}^{2} B_{e}+V_{k} V_{m} B_{e} \cos \left(\delta_{k}-\delta_{m}\right)-Q_{m}=0 \\
B_{e}-B_{e}(\propto)=0 \\
\left(P^{2}+Q_{k}^{2}\right)^{\frac{1}{2}}-I V_{k}=0
\end{array}
$$

where $\mathrm{x}_{c}$ and $\mathrm{f}\left(x_{c}, \propto, I, I_{\text {ref }}\right)$ stand for the internal control system variables and equations. $x_{c}$ is the constant reactance TCSC model, $a$ is the firing angle TCSC model, $B$ is the series susceptance of the TCSC, $\mathrm{V}_{\mathrm{k}}$ and $\mathrm{V}_{\mathrm{m}}$ are the terminal voltages of controller, $\delta_{k}$ and $\delta_{m}$ are the magnitudes of the angles at the controller terminals, $Q_{k}$ and $Q_{m}$ are the reactive power injections at both controller terminals, $\mathrm{P}$ and I are the active power and current flowing through the controller respectively, $\mathrm{B}_{\mathrm{e}}$ is given as [14].

$$
\begin{aligned}
B_{e}(\propto)=\pi\left(k_{x}^{4}-\right. & \left.2 k_{x}^{2}+1\right) \cos k_{x}(\pi-\propto) \\
& +\left[X _ { C } \left(\pi k_{x}^{4} \cos k_{x}(\pi-\propto)\right.\right. \\
& -\pi \cos \left(k_{x}-\propto\right)-2 k_{x}^{4} \\
& \propto \cos k_{x}(\pi-\propto)
\end{aligned}
$$

Where

$$
\left.k_{x} \text { (Percentage of compensation of TCSC }\right)=\left(\frac{x_{C}}{x_{L}}\right)^{\frac{1}{2}}
$$

For an impedance control model with no droop, which yields the simplest set of steady state equations from the numerical point of view, the power flow equations for the TCSC are

$$
\begin{gathered}
B_{e}-B_{r e f}=0 \\
P+V_{k} V_{m} B_{e} \sin \left(\delta_{k}-\delta_{m}\right)=0 \\
-V_{k}^{2} B_{e}+V_{k} V_{m} B_{e} \cos \left(\delta_{k}-\delta_{m}\right)-Q_{k}=0 \\
B_{e}-B_{e}(\propto)=0 \\
\left(P^{2}+Q_{k}^{2}\right)^{\frac{1}{2}}-I V_{k}=0
\end{gathered}
$$

\subsection{SSSC}

The functional model of SSSC is shown in Figure 2. The SSSC is a series voltage source equipped with a source of energy in the DC link that can supply or absorb the reactive and active power to or from the line [16]. 


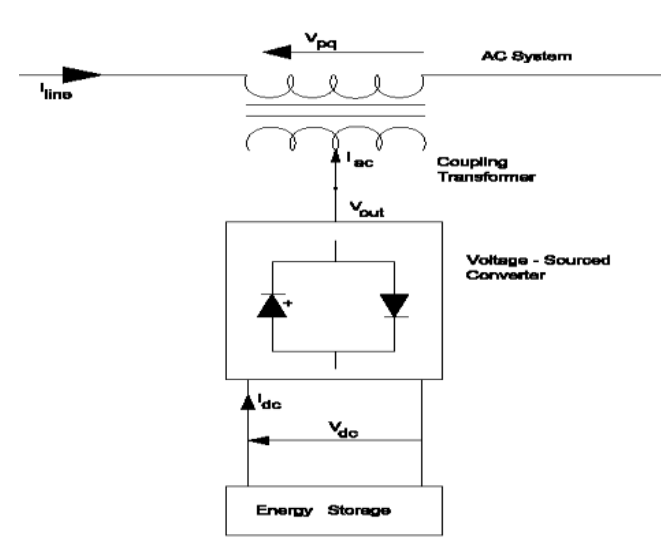

Figure 2: Functional Model of Static Synchronous Series Compensator [15]

\subsubsection{Active power flow control}

The active power flow constraint is as follows [17]

$$
P_{j i}-P_{j i}^{r e f}=0
$$

where $P_{j i}^{r e f}$ is the specified active power flow control reference along the line between bus $j$ and $i$, and $P_{j i}$ is the active power flow given as;

$$
\begin{aligned}
P_{i j}=V_{i}^{2} g_{i i}-V_{i} & V_{j}\left(g_{i j} \cos \theta_{i j}+b_{i j} \sin \theta_{i j}\right) \\
& -V_{i} V_{s e}\left(g_{i j} \cos \left(\theta_{i}-\theta_{s e}\right)\right. \\
& \left.+b_{i j} \sin \left(\theta_{i}-\theta_{s e}\right)\right)
\end{aligned}
$$

Where $\quad g_{i j}+j b_{i j}=\frac{1}{z_{s e}}, g_{i i}=g_{i j}, b_{i i}=b_{i j}, g_{j j}=$ $g_{i j}, b_{j j}=. b_{i j} ; \mathrm{V}_{\mathrm{se}}$ and $\mathrm{Z}_{\mathrm{se}}$ are the voltage source and impedance sources respectively.

\subsubsection{Reactive power flow control}

The reactive power flow constraint is as follows;

$$
Q_{j i}-Q_{j i}^{r e f}=0
$$

where $Q_{j i}^{r e f}$ is the specified reactive power flow control reference, $Q_{j i}$ is the SSSC branch reactive power flows and

$$
\begin{aligned}
Q_{i j}=V_{i}^{2} b_{i j}-V_{i} & V_{j}\left(g_{i j} \sin \theta_{i j}-b_{i j} \cos \theta_{i j}\right) \\
& -V_{i} V_{s e}\left(g_{i j} \sin \left(\theta_{i}-\theta_{s e}\right)\right. \\
& \left.-b_{i j} \cos \left(\theta_{i}-\theta_{s e}\right)\right)
\end{aligned}
$$

\subsubsection{Bus Voltage Control}

The bus voltage control constraints is given as;

$$
V_{i}-V_{i}^{r e f}=0, V_{j}^{r e f}-V_{j}=0
$$

where $V_{i}^{\text {ref }}$ and $V_{j}^{\text {ref }}$ are the bus voltage references.

\subsubsection{Reactance Control}

In this control function, $V_{s e}$ is regulated to control equivalent reactance of the SSSC to a specified reactance reference;

$$
I_{s e}=I_{j i}=\sqrt{\frac{\mathrm{V}_{\mathrm{i}}^{2}+\mathrm{V}_{\mathrm{se}}^{2}+\mathrm{V}_{\mathrm{j}}^{2}-2 \mathrm{~V}_{\mathrm{i}} \mathrm{V}_{\mathrm{se}} \cos \left(\theta_{\mathrm{i}}-\theta_{\mathrm{se}}\right)+2 \mathrm{~V}_{\mathrm{i}} \mathrm{V}_{\mathrm{se}} \cos \left(\theta_{\mathrm{i}}-\theta_{\mathrm{se}}\right)-2 \mathrm{~V}_{\mathrm{i}} \mathrm{V}_{\mathrm{j}} \cos \left(\theta_{\mathrm{i}}-\theta_{\mathrm{se}}\right.}{\left|\mathrm{Z}_{\mathrm{se}}\right|}}
$$

where $X_{c}^{\text {ref }}$ is the reference reactance, $X_{c}$ is the function of state variables $V_{i}, V_{j}$ and $V_{s e}$. The objective function can be written as

where $x=\left[\theta_{i}, \theta_{j}, \theta_{s e}, V_{i}, V_{j}, V_{s e}\right]^{t}$

\subsubsection{Voltage and Current Constraints of the SSSC}

The equivalent voltage injection $V_{\text {se }}$ bound constraint is

$$
0 \leq V_{s e} \leq V_{s e}^{\max }
$$

where $V_{s e}^{\max }$ is the voltage rating of $\mathrm{V}_{\mathrm{se}}$, which may be constant or change slightly with the changes in the DC bus voltage, depending on the inverter design.

The current through each series converter should be within its current rating:

$$
I_{s e} \leq I_{s e}^{\max }
$$

where $I_{s e}^{\max }$ is the current rating of the series converter. But $I_{s e}=I_{s e}<\theta_{s e}=\frac{V_{i}-V_{s e}-V_{j}}{Z_{s e}}$, the actual current magnitude through the SSSC can be obtained as (22).

\section{MODELING OF THE NIGERIAN 48-BUS SYSTEM}

Using PSAT software in MATLAB, modeling of the systems shown in Figures 3 and 4 were achieved with the optimal placement of TCSC and SSSC on line 3-4 in the 48-bus system of Nigeria which comprises of 16 PV generators for load flow studies, 79 transmission lines and 32 load buses obtained from the Transmission Company of Nigeria, National Control Centre, Oshogbo.

Kano transmission station at bus 4 had experienced very low voltage during load flow calculations, hence the need to place the FACTS devices on line 3-4. Tables 1 and 2 show all the data for the 48 buses and transmission lines respectively.

\section{SIMULATION RESULTS OF NIGERIAN 48-BUS SYSTEM}

The steady state performances of TCSC and SSSC are here presented by the outcome of the voltage magnitude profile at the buses, the real and reactive power loses experienced at the transmission lines. 
Newton Raphson power flow simulations were carried out on the modeled Nigerian 48-Bus systems of Figures 3 and 4 first without FACTS and thereafter with TCSC and SSSC. In 0.156 second, the systems without FACTS and with SSSC converges at 2.9437 e-09 p.u. and 1.6667 e-10 p.u. respectively after 4 iterations while the system with TCSC converges at $9.6232 \mathrm{e}-11$ p.u. after 4 iterations in 0.687 second. The summaries of the results of the power flow are shown in Tables 3 and 6 . Table 4 shows the results of the continuation power flow (CPF) where the load at the buses were increased successively until the critical loading parameter was reached. Following the successful completion of power flow and CPF simulations for the
Nigerian 48-bus system, voltage stability sensitivity factors (VSSF) were computed for all the load buses as displayed in Table 5. The result of the CPF is completed in 2.0922 seconds with maximum loading parameter $\left(\lambda_{\max }\right)$ equaling 3.1887. Taking a look at Table 4, it can be observed that buses 3(Kaduna), 4(Kano), 6(Makurdi), 9(Jos), 13(Osogbo), 22(Ugwuaji) and 28(Ayede) were found to be very weak buses with voltages well below 0.800 p.u. Bus 4(Kano) is noticed to be the weakest bus with voltage magnitude of 0.36128 p.u. From Table 5 (VSSF), Bus 4(Kano) is also seen to have the highest factor of 0.57724 and therefore, the weakest bus and most suitable for the installation of TCSC and SSSC.

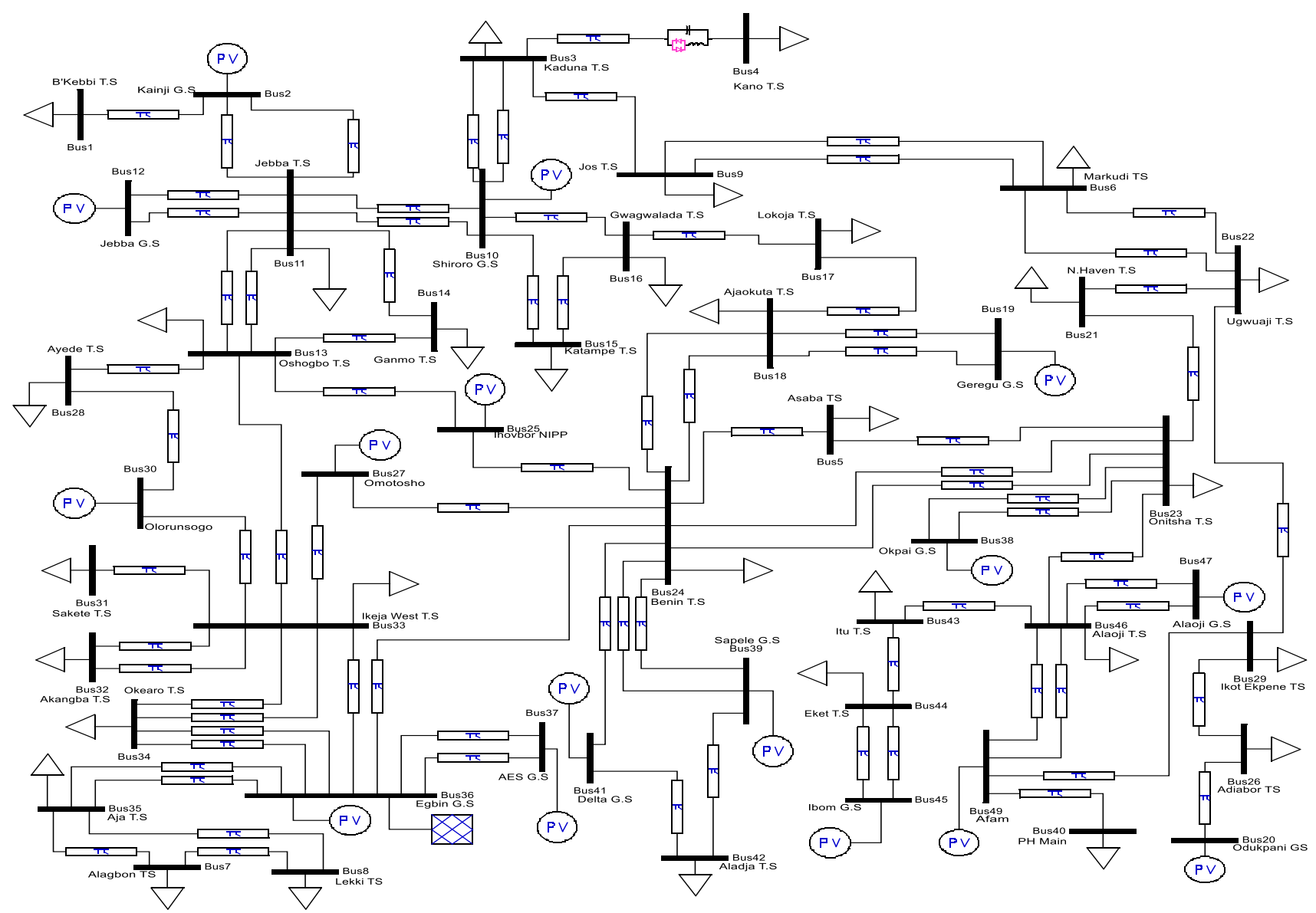

Figure 3: Nigerian 48-bus system with TCSC placed on line 3-4 


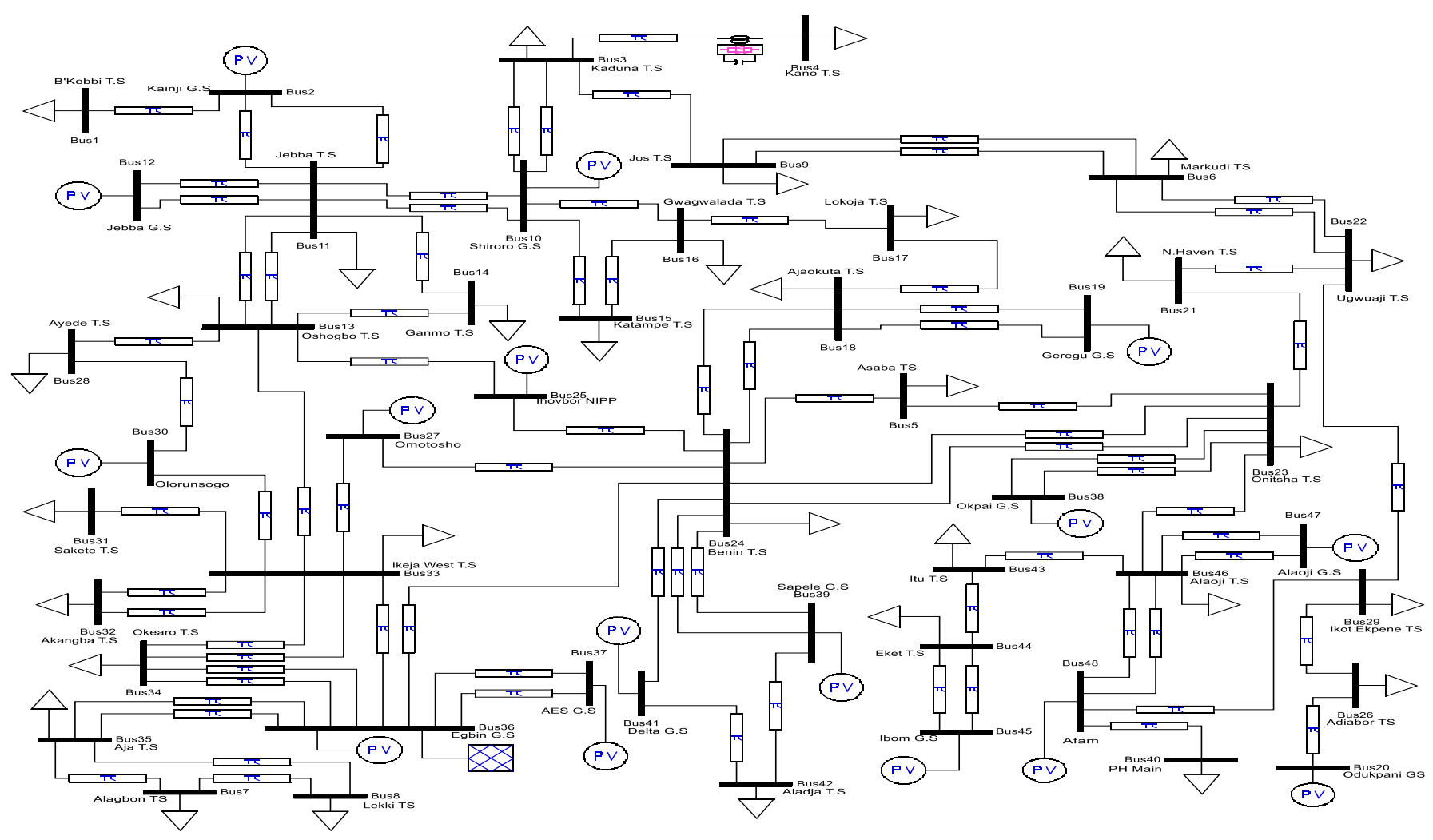

Figure 4: Nigerian 48-bus system with SSSC placed on line 3-4

Table 1: Bus data for Nigerian 48-bus system

\begin{tabular}{ccccccccc}
\hline & & NOM & ACTUAL & VOLT & \multicolumn{2}{c}{ LOAD } & \multicolumn{2}{c}{ GENERATION } \\
\cline { 6 - 9 } BUS & BUS & VOLT & VOLT & MAG. & MW & MVAR & MW & MVAR \\
NO & NAME & $(\mathrm{kV})$ & $(\mathrm{kV})$ & $(\mathrm{PU})$ & & & & \\
\hline 1 & Birnin Kebbi & 330 & 326 & 0.988 & 100 & 62 & - & - \\
2 & Kainji GS & 330 & 330 & 1.0 & - & - & 492 & 158 \\
3 & Kaduna & 330 & 315 & 0.956 & 120 & 90 & - & - \\
4 & Kano & 330 & 300 & 0.909 & 41 & 26 & - & - \\
5 & Asaba & 330 & 305 & 0.924 & 80 & 59 & - & - \\
6 & Markudi & 330 & 310 & 0.939 & 100 & 60 & - & - \\
7 & Alagbon & 330 & 320 & 0.97 & 70 & 43 & - & - \\
8 & Lekki & 330 & 300 & 0.909 & 110 & 78 & - & - \\
9 & Jos & 330 & 310 & 0.939 & 160 & 70 & - & - \\
10 & Shiroro GS & 330 & 330 & 1.0 & - & - & 500 & 115 \\
11 & Jebba & 330 & 326 & 0.988 & 260 & 195 & - & - \\
12 & Jebba GS & 330 & 330 & 1.0 & - & - & 403 & 190 \\
13 & Oshogbo & 330 & 330 & 1.0 & 127 & 95 & - & - \\
14 & Ganmo & 330 & 328 & 0.994 & 100 & 75 & - & - \\
15 & Katampe & 330 & 330 & 1.0 & 303 & 227 & - & - \\
16 & Gwagwalada & 330 & 315 & 0.956 & 220 & 165 & - & - \\
17 & Lokoja & 330 & 320 & 0.97 & 120 & 90 & - & - \\
18 & Ajaokuta & 330 & 320 & 0.97 & 120 & 90 & - & - \\
19 & Geregu GS & 330 & 330 & 1.0 & - & - & 531 & 230 \\
20 & Odukpani GS & 330 & 330 & 1.0 & - & - & 260 & 121 \\
21 & New Heaven & 330 & 326 & 0.988 & 196 & 147 & - & - \\
22 & Ugwuaji & 330 & 328 & 0.994 & 175 & 131 & - & -
\end{tabular}




\begin{tabular}{lcccccccc}
\hline & & & & & & \\
NOM & ACTUAL & VOLT & \multicolumn{2}{c}{ LOAD } & \multicolumn{2}{c}{ GENERATION } \\
\cline { 6 - 9 } NOS & BUS & VOLT & VOLT & MAG. & MW & MVAR & MW & MVAR \\
\hline 23 & NAME & $(\mathrm{kV})$ & $(\mathrm{kV})$ & $(\mathrm{PU})$ & & & & - \\
24 & Onitsha & 330 & 324 & 0.982 & 100 & 75 & - & - \\
25 & Benin & 330 & 328 & 0.994 & 144 & 108 & - & - \\
26 & Ihovbor GS & 330 & 330 & 1.0 & - & - & 117 & 71 \\
27 & Adiabor & 330 & 320 & 0.97 & 90 & 48 & - & - \\
28 & Omotosho GS & 330 & 330 & 1.0 & - & - & 165 & 73 \\
29 & Ayede & 330 & 320 & 0.97 & 190 & 151 & - & - \\
30 & Ikot Ekpene & 330 & 313 & 0.948 & 165 & 74 & - & - \\
31 & Olorunsogo GS & 330 & 330 & 1.0 & - & - & 196 & 124 \\
32 & Sakete & 330 & 300 & 0.909 & 225 & 190 & - & - \\
33 & Akangba & 330 & 330 & 1.0 & 203 & 152 & - & - \\
34 & Ikeja West & 330 & 330 & 1.0 & 847 & 635 & - & - \\
35 & Okearo & 330 & 330 & 1.0 & 120 & 90 & - & - \\
36 & Aja & 330 & 330 & 1.0 & 120 & 90 & - & - \\
37 & Egbin GS & 330 & 330 & 1.0 & - & - & 513 & 240 \\
38 & AES GS & 330 & 330 & 1.0 & - & - & 245 & 195 \\
39 & Okpai GS & 330 & 330 & 1.0 & - & - & 466 & 200 \\
40 & Sapele GS & 330 & 330 & 1.0 & - & - & 178 & 79 \\
41 & PH Main & 330 & 300 & 0.909 & 280 & 140 & - & - \\
42 & Delta GS & 330 & 330 & 1.0 & - & - & 341 & 115 \\
43 & Aladja & 330 & 325 & 0.985 & 210 & 158 & - & - \\
44 & Itu & 330 & 330 & 1.0 & 199 & 91 & - & - \\
45 & Eket & 330 & 328 & 0.994 & 200 & 147 & - & - \\
46 & Ibom GS & 330 & 330 & 1.0 & - & - & 31 & 12 \\
47 & Alaoji & 330 & 320 & 0.97 & 240 & 100 & - & - \\
48 & Alaoji GS & 330 & 330 & 1.0 & - & - & 250 & 117 \\
\hline & Afam GS & 330 & 321 & 0.973 & - & - & 700 & 506 \\
\hline
\end{tabular}

Source [18]

\begin{tabular}{|c|c|c|c|c|c|}
\hline $\mathrm{S} / \mathrm{N}$ & $\begin{array}{c}\text { FROM } \\
\text { BUS }\end{array}$ & $\begin{array}{c}\text { TO } \\
\text { BUS }\end{array}$ & $R(P U)$ & $X(P U)$ & $\begin{array}{l}\text { 1/2 B } \\
\text { (PU) }\end{array}$ \\
\hline 1 & 1 & 2 & 0.0011019 & 0.0142241 & 0.064172 \\
\hline 2 & 2 & 11 & 0.0039008 & 0.0246198 & 0.036385 \\
\hline 3 & 2 & 11 & 0.0039008 & 0.0246198 & 0.036385 \\
\hline 4 & 3 & 4 & 0.0030370 & 0.0300082 & 0.030295 \\
\hline 5 & 3 & 9 & 0.0022193 & 0.0115139 & 0.076896 \\
\hline 6 & 3 & 10 & 0.0094380 & 0.0391791 & 0.022152 \\
\hline 7 & 3 & 10 & 0.0094380 & 0.0391791 & 0.022152 \\
\hline 8 & 5 & 23 & 0.0017171 & 0.0145744 & 0.062143 \\
\hline 9 & 5 & 24 & 0.0031889 & 0.0270666 & 0.003248 \\
\hline 10 & 6 & 9 & 0.0053470 & 0.0195760 & 0.043652 \\
\hline 11 & 6 & 9 & 0.0053470 & 0.0195760 & 0.043652 \\
\hline 12 & 6 & 22 & 0.0064850 & 0.0553970 & 0.016352 \\
\hline 13 & 6 & 22 & 0.0064850 & 0.0553970 & 0.016352 \\
\hline 14 & 7 & 8 & 0.0091700 & 0.0292470 & 0.028587 \\
\hline 15 & 7 & 35 & 0.0009410 & 0.0072340 & 0.124826 \\
\hline 16 & 8 & 35 & 0.0049670 & 0.0157480 & 0.053035 \\
\hline 17 & 10 & 11 & 0.0087383 & 0.0741635 & 0.012212 \\
\hline 18 & 10 & 11 & 0.0087383 & 0.0741635 & 0.012212 \\
\hline 19 & 10 & 15 & 0.0048872 & 0.0106557 & 0.071355 \\
\hline 20 & 10 & 16 & 0.0052820 & 0.0106200 & 0.069319 \\
\hline
\end{tabular}

\begin{tabular}{cccccc}
\hline S/N & $\begin{array}{c}\text { FROM } \\
\text { BUS }\end{array}$ & $\begin{array}{c}\text { TO } \\
\text { BUS }\end{array}$ & R (PU) & X(PU) & $\begin{array}{c}1 / 2 \mathrm{~B} \\
(\mathrm{PU})\end{array}$ \\
\hline 21 & 11 & 12 & 0.0002890 & 0.0022300 & 0.404978 \\
22 & 11 & 12 & 0.0002890 & 0.0022300 & 0.404978 \\
23 & 11 & 13 & 0.0056226 & 0.0477199 & 0.018980 \\
24 & 11 & 13 & 0.0056226 & 0.0477199 & 0.018980 \\
25 & 11 & 14 & 0.0039390 & 0.0133430 & 0.063304 \\
26 & 13 & 14 & 0.0016834 & 0.0142860 & 0.063398 \\
27 & 13 & 25 & 0.0089890 & 0.0762910 & 0.011872 \\
28 & 13 & 28 & 0.0041185 & 0.0349541 & 0.025911 \\
29 & 13 & 33 & 0.0089532 & 0.0759871 & 0.011919 \\
30 & 15 & 16 & 0.0016050 & 0.0300600 & 0.002826 \\
31 & 16 & 17 & 0.0043170 & 0.0015800 & 0.068655 \\
32 & 17 & 18 & 0.0017150 & 0.0131860 & 0.068482 \\
33 & 18 & 19 & 0.0000540 & 0.0005100 & 1.780575 \\
34 & 18 & 19 & 0.0000540 & 0.0005100 & 1.780575 \\
35 & 18 & 24 & 0.0070551 & 0.0542562 & 0.016643 \\
36 & 18 & 24 & 0.0070551 & 0.0542562 & 0.016643 \\
37 & 20 & 26 & 0.0005770 & 0.0048910 & 0.185171 \\
38 & 21 & 22 & 0.0002530 & 0.0019480 & 0.463574 \\
39 & 21 & 23 & 0.0002380 & 0.0004321 & 1.604128 \\
40 & 22 & 29 & 0.0059210 & 0.0219100 & 0.039059 \\
41 & 23 & 24 & 0.0049063 & 0.0416410 & 0.021750
\end{tabular}




\begin{tabular}{|c|c|c|c|c|c|}
\hline$S / N$ & $\begin{array}{c}\text { FROM } \\
\text { BUS }\end{array}$ & $\begin{array}{c}\text { TO } \\
\text { BUS }\end{array}$ & R (PU) & $X(P U)$ & $\begin{array}{l}1 / 2 \mathrm{~B} \\
(\mathrm{PU})\end{array}$ \\
\hline 42 & 23 & 24 & 0.0049063 & 0.0416410 & 0.021750 \\
\hline 43 & 23 & 38 & 0.0021708 & 0.0166942 & 0.082468 \\
\hline 44 & 23 & 38 & 0.0021708 & 0.0166942 & 0.082468 \\
\hline 45 & 23 & 46 & 0.0049422 & 0.0419449 & 0.021593 \\
\hline 46 & 24 & 25 & 0.0008990 & 0.0076200 & 0.118854 \\
\hline 47 & 24 & 27 & 0.0018264 & 0.0155014 & 0.059169 \\
\hline 48 & 24 & 36 & 0.0071025 & 0.0607897 & 0.014902 \\
\hline 49 & 24 & 39 & 0.0018090 & 0.0139118 & 0.064909 \\
\hline 50 & 24 & 39 & 0.0018090 & 0.0139118 & 0.064909 \\
\hline 51 & 24 & 41 & 0.0014683 & 0.0124619 & 0.072678 \\
\hline 52 & 26 & 29 & 0.0021880 & 0.0185740 & 0.048762 \\
\hline 53 & 27 & 33 & 0.0028640 & 0.0243150 & 0.037249 \\
\hline 54 & 28 & 30 & 0.0021488 & 0.0182369 & 0.049663 \\
\hline 55 & 29 & 48 & 0.0023030 & 0.0195500 & 0.046328 \\
\hline 56 & 30 & 33 & 0.0027566 & 0.0234030 & 0.039234 \\
\hline 57 & 31 & 33 & 0.0025069 & 0.0212764 & 0.042568 \\
\hline 58 & 32 & 33 & 0.0006151 & 0.0047300 & 0.190909 \\
\hline 59 & 32 & 33 & 0.0006151 & 0.0047300 & 0.190909 \\
\hline 60 & 33 & 34 & 0.0006450 & 0.0054710 & 0.165543 \\
\hline 61 & 33 & 34 & 0.0006450 & 0.0054710 & 0.165543 \\
\hline 62 & 33 & 36 & 0.0006450 & 0.0054710 & 0.165543 \\
\hline 63 & 34 & 36 & 0.0006450 & 0.0054710 & 0.165543 \\
\hline 64 & 34 & 36 & 0.0006450 & 0.0054710 & 0.165543 \\
\hline 65 & 35 & 36 & 0.0005014 & 0.0042553 & 0.939551 \\
\hline 66 & 35 & 36 & 0.0005014 & 0.0042553 & 0.939551 \\
\hline 67 & 36 & 37 & 0.0071625 & 0.0607897 & 0.014899 \\
\hline 68 & 36 & 37 & 0.0071625 & 0.0607897 & 0.014899 \\
\hline 69 & 39 & 42 & 0.0022562 & 0.0191488 & 0.047298 \\
\hline 70 & 40 & 48 & 0.0009045 & 0.0069559 & 0.129819 \\
\hline 71 & 41 & 42 & 0.0011460 & 0.0097264 & 0.093118 \\
\hline 72 & 43 & 44 & 0.0009045 & 0.0069559 & 0.129819 \\
\hline 73 & 43 & 46 & 0.0009045 & 0.0069559 & 0.129819 \\
\hline 74 & 44 & 45 & 0.0009045 & 0.0069559 & 0.129819 \\
\hline 75 & 44 & 45 & 0.0009045 & 0.0069559 & 0.129819 \\
\hline 76 & 46 & 47 & 0.0049422 & 0.0419449 & 0.021593 \\
\hline 77 & 46 & 47 & 0.0049422 & 0.0419449 & 0.021593 \\
\hline 78 & 46 & 48 & 0.0009045 & 0.0069559 & 0.129819 \\
\hline 79 & 46 & 48 & 0.0009045 & 0.0069559 & 0.129819 \\
\hline
\end{tabular}

Table 3: Power flow results of Nigerian 48-Bus system without FACTS

\begin{tabular}{cccccc}
\hline $\begin{array}{c}\text { BUS } \\
\text { NO }\end{array}$ & BUS NAME & $\begin{array}{c}\text { VOLTAGE } \\
(\mathrm{pu})\end{array}$ & $\begin{array}{c}\text { PHASE } \\
\text { ANGLE } \\
(\mathrm{rad})\end{array}$ & $\begin{array}{c}\text { REAL } \\
\text { POWER } \\
(\mathrm{pu})\end{array}$ & $\begin{array}{c}\text { REACTIVE } \\
\text { POWER } \\
(\mathrm{pu})\end{array}$ \\
\hline 1 & Birnin Kebbi & 0.9899 & 0.1273 & -1 & -0.62 \\
2 & Kainji GS & 1.0 & 0.1410 & 4.92 & 0.5016 \\
$\mathbf{3}$ & Kaduna & $\mathbf{0 . 9 4 8 2}$ & $-\mathbf{0 . 0 0 2 0}$ & -1.2 & -0.9 \\
$\mathbf{4}$ & Kano & $\mathbf{0 . 9 3 8 5}$ & $-\mathbf{0 . 0 1 4 9}$ & $-\mathbf{0 . 4 1}$ & $-\mathbf{0 . 2 6}$ \\
$\mathbf{5}$ & Asaba & 0.9745 & 0.0369 & -0.8 & -0.59 \\
$\mathbf{6}$ & Makurdi & $\mathbf{0 . 9 4 0 5}$ & $-\mathbf{0 . 0 1 0 7}$ & -1.0 & -0.6 \\
7 & Alagbon & 1.0225 & -0.0113 & -0.7 & -0.43 \\
8 & Lekki & 1.0146 & -0.0160 & -1.1 & -0.78 \\
$\mathbf{9}$ & Jos & $\mathbf{0 . 9 3 8 1}$ & -0.0154 & -1.6 & -0.7 \\
10 & Shiroro GS & 1.0 & 0.0463 & 5.0 & 6.3177 \\
11 & Jebba & 0.9955 & 0.0923 & -2.6 & -1.95 \\
12 & Jebba GS & 1.0 & 0.0962 & 4.03 & 3.77 \\
13 & Oshogbo & 0.9716 & 0.0477 & -1.27 & -0.95 \\
14 & Ganmo & 0.9747 & 0.0655 & -1.0 & -0.75 \\
15 & Katampe & 0.969 & 0.0382 & -3.03 & -2.27
\end{tabular}

\begin{tabular}{|c|c|c|c|c|c|}
\hline $\begin{array}{l}\text { BUS } \\
\text { NO }\end{array}$ & BUS NAME & $\begin{array}{c}\text { VOLTAGE } \\
(\mathrm{pu})\end{array}$ & $\begin{array}{c}\text { PHASE } \\
\text { ANGLE } \\
\text { (rad) } \\
\end{array}$ & $\begin{array}{c}\text { REAL } \\
\text { POWER } \\
(\mathrm{pu}) \\
\end{array}$ & $\begin{array}{c}\text { REACTIVE } \\
\text { POWER } \\
\text { (pu) }\end{array}$ \\
\hline 16 & Gwagwalada & 0.9713 & 0.0383 & -2.2 & -1.65 \\
\hline 17 & Lokoja & 0.9800 & 0.0413 & -1.2 & -0.9 \\
\hline 18 & Ajaokuta & 0.9993 & 0.0819 & -1.2 & -0.9 \\
\hline 19 & Geregu GS & 1.0 & 0.0832 & 5.31 & 2.0461 \\
\hline 20 & $\begin{array}{l}\text { Odukpani } \\
\text { GS }\end{array}$ & 1.0 & 0.0842 & 2.6 & 0.9802 \\
\hline 21 & New heaven & 0.9709 & 0.0361 & -1.96 & -1.47 \\
\hline 22 & Ugwuaji & 0.9670 & 0.031 & -1.75 & -1.31 \\
\hline 23 & Onitsha & 0.9734 & 0.0374 & -1.0 & -0.75 \\
\hline 24 & Benin & 0.9956 & 0.0562 & -1.44 & -1.08 \\
\hline 25 & Ihovbor GS & 1,0 & 0.0629 & 1.166 & 0.8111 \\
\hline 26 & Adiabor & 0.9938 & 0.0719 & -0.9 & -0.48 \\
\hline 27 & $\begin{array}{l}\text { Omotosho } \\
\text { GS }\end{array}$ & 1.006 & 0.0412 & 1.65 & 0.9512 \\
\hline 28 & Ayede & 0.9483 & 0.0036 & -1.9 & -1.51 \\
\hline 29 & Ikot Ekpene & 0.9819 & 0.0407 & -1.65 & -0.74 \\
\hline 30 & $\begin{array}{c}\text { Olorunsogo } \\
\text { GS }\end{array}$ & 0.97 & 0.0148 & 1.96 & -0.1507 \\
\hline 31 & Sakete & 0.9469 & -0.0653 & -2.25 & -1.9 \\
\hline 32 & Akangba & 0.9923 & -0.0240 & -2.03 & -1.52 \\
\hline 33 & Ikeja West & 0.9966 & -0.0196 & -8.47 & -6.35 \\
\hline 34 & Okearo & 1.0133 & -0.0111 & -1.2 & -0.9 \\
\hline 35 & Aja & 1.0278 & -0.0055 & -1.2 & -0.9 \\
\hline 36 & Egbin GS & 1.033 & 0.0 & 9.2005 & 17.6746 \\
\hline 37 & AES GS & 1.0 & 0.0766 & 2.452 & -1.2759 \\
\hline 38 & Okpai GS & 1.0 & 0.0744 & 4.66 & 2.6555 \\
\hline 39 & Sapele GS & 1.0 & 0.0658 & 1.78 & 0.9291 \\
\hline 40 & PH Main & 0.9874 & 0.0306 & -2.8 & -1.4 \\
\hline 41 & Delta GS & 1.003 & 0.0762 & 3.41 & 1.5599 \\
\hline 42 & Aladja & 0.99 & 0.0603 & -2.1 & -1.58 \\
\hline 43 & Itu & 0.9878 & 0.0109 & -1.99 & -0.91 \\
\hline 44 & Eket & 0.9919 & -0.0019 & -2.0 & -1.47 \\
\hline 45 & Ibom GS & 1.0 & -0.0019 & 0.305 & 2.2952 \\
\hline 46 & Alaoji & 0.9924 & 0.0370 & -2.4 & -1.0 \\
\hline 47 & Alaoji GS & 1.0 & 0.0895 & 2.5 & 0.1307 \\
\hline 48 & Afam GS & 1.0 & 0.0491 & 7.0 & 4.0565 \\
\hline
\end{tabular}

Table 4: Continuation power flow (CPF) results of Nigerian 48-bus system

\begin{tabular}{cccccc}
\hline $\begin{array}{c}\text { BUS } \\
\text { NO }\end{array}$ & BUS NAME & $\begin{array}{c}\text { VOLTAGE } \\
(\mathrm{pu})\end{array}$ & $\begin{array}{c}\text { PHASE } \\
\text { ANGLE } \\
(\mathrm{deg})\end{array}$ & $\begin{array}{c}\text { REAL } \\
\text { POWER } \\
(\mathrm{pu})\end{array}$ & $\begin{array}{c}\text { REACTIVE } \\
\text { POWER } \\
(\mathrm{pu})\end{array}$ \\
\hline $\mathbf{1}$ & Birnin Kebbi & 0.9671 & 80.9923 & -3.1179 & -1.9331 \\
$\mathbf{2}$ & Kainji GS & 1.0000 & 83.4945 & 15.3402 & 3.9795 \\
$\mathbf{3}$ & Kaduna & $\mathbf{0 . 4 5 0 5}$ & $\mathbf{5 2 . 4 5 7 8}$ & $\mathbf{- 3 . 7 4 1 5}$ & $\mathbf{- 2 . 8 0 6 1}$ \\
$\mathbf{4}$ & Kano & $\mathbf{0 . 3 6 1 3}$ & 39.714 & $-\mathbf{1 . 2 7 8 4}$ & $-\mathbf{- 0 . 8 1 0 7}$ \\
5 & Asaba & 0.8244 & 62.0247 & 0.0000 & 0.0000 \\
$\mathbf{6}$ & Makurdi & $\mathbf{0 . 4 5 2 7}$ & $\mathbf{4 8 . 3 5 8 3}$ & $\mathbf{- 3 . 1 1 7 9}$ & -1.8708 \\
$\mathbf{7}$ & Alagbon & 0.9954 & -2.301 & -2.1826 & -1.3407 \\
8 & Lekki & 0.9692 & -3.1918 & -3.4297 & -2.432 \\
$\mathbf{9}$ & Jos & $\mathbf{0 . 4 5 0 7}$ & $\mathbf{5 0 . 9 5 6 7}$ & $\mathbf{0 . 0 0 0 0}$ & $\mathbf{0 . 0 0 0 0}$ \\
10 & Shiroro GS & 1.0000 & 75.7232 & 15.5896 & 20.4486 \\
11 & Jebba & 0.9533 & 65.5731 & -8.1066 & -6.0800 \\
12 & Jebba GS & 1.0000 & 66.9195 & 12.5652 & 19.4490 \\
$\mathbf{1 3}$ & Oshogbo & $\mathbf{0 . 7 6 6 6}$ & $\mathbf{4 2 . 3 9 0 7}$ & $\mathbf{- 3 . 9 5 9 8}$ & $\mathbf{- 2 . 9 6 2 0}$ \\
14 & Ganmo & 0.8297 & 56.0152 & 0.0000 & 0.0000 \\
15 & Katampe & 0.9435 & 75.0664 & -9.4473 & -7.0777 \\
16 & Gwagwalada & 0.9562 & 74.8802 & 0.0000 & 0.0000 \\
17 & Lokoja & 0.9763 & 75.0931 & 0.0000 & 0.0000 \\
18 & Ajaokuta & 0.9957 & 78.3379 & -3.7415 & -2.8061 \\
19 & Geregu GS & 1.0000 & 78.8026 & 16.5562 & 6.8176 \\
20 & Odukpani & 1.0000 & 92.7037 & 8.1066 & 3.8203 \\
& GS & & & & \\
& & & & &
\end{tabular}




\begin{tabular}{|c|c|c|c|c|c|}
\hline $\begin{array}{l}\text { BUS } \\
\text { NO }\end{array}$ & BUS NAME & $\begin{array}{c}\text { VOLTAGE } \\
(\mathrm{pu})\end{array}$ & $\begin{array}{l}\text { PHASE } \\
\text { ANGLE } \\
\text { (deg) }\end{array}$ & $\begin{array}{c}\text { REAL } \\
\text { POWER } \\
\text { (pu) }\end{array}$ & $\begin{array}{c}\text { REACTIVE } \\
\text { POWER } \\
\text { (pu) }\end{array}$ \\
\hline 21 & New heaven & 0.8002 & 69.9862 & -6.1111 & -4.5834 \\
\hline 22 & Ugwuaji & 0.7932 & 70.6709 & 0.0000 & 0.0000 \\
\hline 23 & Onitsha & 0.8053 & 69.9203 & -3.1179 & -2.3384 \\
\hline 24 & Benin & 0.8980 & 48.7994 & -4.4898 & -3.3674 \\
\hline 25 & Ihovbor GS & 1.0000 & 49.0774 & 3.6355 & 16.0759 \\
\hline 26 & Adiabor & 0.9774 & 90.508 & 0.0000 & 0.0000 \\
\hline 27 & $\begin{array}{c}\text { Omotosho } \\
\text { GS }\end{array}$ & 0.8155 & 30.2182 & 0.0000 & 0.0000 \\
\hline 28 & Ayede & 0.7863 & 14.6255 & -5.9241 & -4.7081 \\
\hline 29 & Ikot Ekpene & 0.9059 & 81.2653 & -5.1446 & -2.3073 \\
\hline 30 & $\begin{array}{c}\text { Olorunsogo } \\
\text { GS }\end{array}$ & 0.9700 & 10.9736 & 6.1111 & 13.4839 \\
\hline 31 & Sakete & 0.8823 & -0.3599 & 0.0000 & 0.0000 \\
\hline 32 & Akangba & 0.8508 & -2.423 & -6.3294 & -4.7392 \\
\hline 33 & Ikeja West & 0.8823 & -0.3598 & -26.4088 & -19.7988 \\
\hline 34 & Okearo & 0.9576 & -0.1165 & 0.0000 & 0.0000 \\
\hline 35 & Aja & 1.0129 & -1.2141 & 0.0000 & 0.0000 \\
\hline 36 & Egbin GS & 1.0330 & 0.0000 & 1.7733 & 54.9283 \\
\hline 37 & AES GS & 1.0330 & -0.0002 & 0.0000 & 0.0000 \\
\hline 38 & Okpai GS & 1.0000 & 85.5865 & 14.5295 & 11.5664 \\
\hline 39 & Sapele GS & 0.9313 & 50.8127 & 0.0000 & 0.0000 \\
\hline 40 & PH Main & 0.9583 & 82.5183 & -8.7302 & -4.3651 \\
\hline 41 & Delta GS & 1.0030 & 54.5985 & 10.6321 & 10.1301 \\
\hline 42 & Aladja & 0.9784 & 53.3854 & 0.0000 & 0.0000 \\
\hline 43 & Itu & 0.9309 & 77.3906 & -6.2047 & -2.8373 \\
\hline 44 & Eket & 0.9451 & 74.808 & -6.2359 & -4.5834 \\
\hline 45 & Ibom GS & 1.0000 & 74.7831 & 0.9600 & 7.7669 \\
\hline 46 & Alaoji & 0.9487 & 82.6034 & 0.0000 & 0.0000 \\
\hline 47 & Alaoji GS & 1.0000 & 102.2478 & 7.7948 & 1.6201 \\
\hline 48 & Afam GS & 1.0000 & 85.915 & 21.8255 & 16.0121 \\
\hline
\end{tabular}

Table 5: Voltage stability sensitivity factors (VSSF) of the Nigerian 48Bus system

\begin{tabular}{ccc}
\hline BUS NO & BUS NAME & VOLTAGE SENSITIVITY FACTOR \\
\hline 1 & Birnin Kebbi & 0.0228 \\
2 & Kainji GS & 0.0000 \\
3 & Kaduna & 0.4978 \\
4 & Kano & 0.5772 \\
5 & Asaba & 0.1501 \\
6 & Makurdi & 0.4878 \\
7 & Alagbon & 0.0272 \\
8 & Lekki & 0.0454 \\
9 & Jos & 0.4874 \\
10 & Shiroro GS & 0.0000 \\
11 & Jebba & 0.0423 \\
12 & Jebba GS & 0.0000 \\
13 & Oshogbo & 0.2050 \\
14 & Ganmo & 0.1449 \\
15 & Katampe & 0.0255 \\
16 & Gwagwalada & 0.0151 \\
17 & Lokoja & 0.0037 \\
18 & Ajaokuta & 0.0037 \\
19 & Geregu GS & 0.0000 \\
20 & Odukpani GS & 0.0000 \\
21 & New heaven & 0.1707 \\
22 & Ugwuaji & 0.1738 \\
23 & Onitsha & 0.1682 \\
24 & Benin & 0.0977 \\
25 & Ihovbor GS & 0.0000 \\
26 & Adiabor & 0.0164
\end{tabular}

\begin{tabular}{ccc}
\hline BUS NO & BUS NAME & VOLTAGE SENSITIVITY FACTOR \\
\hline 27 & Omotosho GS & 0.0000 \\
28 & Ayede & 0.1620 \\
29 & Ikot Ekpene & 0.0760 \\
30 & Olorunsogo GS & 0.0000 \\
31 & Sakete & 0.0646 \\
32 & Akangba & 0.1415 \\
33 & Ikeja West & 0.1143 \\
34 & Okearo & 0.0537 \\
35 & Aja & 0.0149 \\
36 & Egbin GS & 0.0000 \\
37 & AES GS & 0.0000 \\
38 & Okpai GS & 0.0000 \\
39 & Sapele GS & 0.0000 \\
40 & PH Main & 0.0291 \\
41 & Delta GS & 0.0000 \\
42 & Aladja & 0.0116 \\
43 & Itu & 0.0570 \\
44 & Eket & 0.0468 \\
45 & Ibom GS & 0.0000 \\
46 & Alaoji & 0.0437 \\
47 & Alaoji GS & 0.0000 \\
48 & Afam GS & 0.0000 \\
\hline
\end{tabular}

Table 6: Voltage profile without and with FACTS devices

\begin{tabular}{|c|c|c|c|c|}
\hline $\begin{array}{l}\text { BUS } \\
\text { NO }\end{array}$ & BUS NAME & $\begin{array}{c}\text { NO } \\
\text { FACTs }\end{array}$ & $\begin{array}{l}\text { WITH } \\
\text { TCSC }\end{array}$ & $\begin{array}{l}\text { WITH } \\
\text { SSSC }\end{array}$ \\
\hline 1 & Birnin Kebbi & 0.9899 & 0.9899 & 0.9899 \\
\hline 2 & Kainji GS & 1.0 & 1.0 & 1.0 \\
\hline 3 & Kaduna & 0.9482 & 0.9821 & 0.9717 \\
\hline 4 & Kano & 0.9385 & 0.9805 & 0.9685 \\
\hline 5 & Asaba & 0.9745 & 0.9771 & 0.976 \\
\hline 6 & Makurdi & 0.9405 & 0.9728 & 0.9645 \\
\hline 7 & Alagbon & 1.0225 & 1.0225 & 1.0225 \\
\hline 8 & Lekki & 1.0146 & 1.0146 & 1.016 \\
\hline 9 & Jos & 0.9381 & 0.9788 & 0.9668 \\
\hline 10 & Shiroro GS & 1.0 & 1.0 & 1.0 \\
\hline 11 & Jebba & 0.9952 & 0.9955 & 0.9954 \\
\hline 12 & Jebba GS & 1.0 & 1.0 & 1.0 \\
\hline 13 & Oshogbo & 0.9716 & 0.9755 & 0.9744 \\
\hline 14 & Ganmo & 0.9747 & 0.9768 & 0.9762 \\
\hline 15 & Katampe & 0.969 & 0.969 & 0.969 \\
\hline 16 & Gwagwalada & 0.9713 & 0.9714 & 0.9713 \\
\hline 17 & Lokoja & 0.98 & 0.9804 & 0.9801 \\
\hline 18 & Ajaokuta & 0.9993 & 0.9994 & 0.9993 \\
\hline 19 & Geregu GS & 1.0 & 1.0 & 1.0 \\
\hline 20 & Odukpani GS & 1.0 & 1.0 & 1.0 \\
\hline 21 & New Heaven & 0.9709 & 0.975 & 0.9732 \\
\hline 22 & Ugwuaji & 0.9670 & 0.9725 & 0.9701 \\
\hline 23 & Onitsha & 0.9734 & 0.9771 & 0.9755 \\
\hline 24 & Benin & 0.9956 & 0.9961 & 0.9959 \\
\hline 25 & Ihovbor GS & 1.0 & 1.0 & 1.0 \\
\hline 26 & Adiabor & 0.9938 & 0.9942 & 0.994 \\
\hline 27 & Omotosho GS & 1.006 & 1.006 & 1.006 \\
\hline 28 & Ayede & 0.9483 & 0.9648 & 0.9603 \\
\hline 29 & Ikotekpene & 0.9819 & 0.9836 & 0.9829 \\
\hline 30 & Olorunsogo GS & 0.97 & 0.97 & 0.97 \\
\hline 31 & Sakete & 0.9469 & 0.9888 & 0.9803 \\
\hline
\end{tabular}




\begin{tabular}{ccccc}
\hline BUS & BUS NAME & NO & WITH & WITH \\
NO & FACTs & TCSC & SSSC \\
\hline 32 & Akangba & 0.9923 & 0.9928 & 0.9927 \\
33 & Ikeja West & 0.9966 & 0.9971 & 0.9969 \\
34 & Okearo & 1.0133 & 1.0136 & 1.0135 \\
35 & Aja & 1.0278 & 1.0278 & 1.0278 \\
36 & Egbin GS & 1.033 & 1.033 & 1.033 \\
37 & AES GS & 1.0 & 1.0 & 1.0 \\
38 & Okpai GS & 1.0 & 1.0 & 1.0 \\
39 & Sapele GS & 1.0 & 1.0 & 1.0 \\
40 & PH Main & 0.9874 & 0.9874 & 0.9874 \\
41 & Delta GS & 1.003 & 1.003 & 1.003 \\
42 & Aladja & 0.99 & 0.99 & 0.99 \\
43 & Itu & 0.9878 & 0.988 & 0.9879 \\
44 & Eket & 0.9919 & 0.9919 & 0.9919 \\
45 & Ibom GS & 1.0 & 1.0 & 1.0 \\
46 & Alaoji & 0.9924 & 0.9926 & 0.9926
\end{tabular}

\begin{tabular}{ccccc}
\hline $\begin{array}{c}\text { BUS } \\
\text { NO }\end{array}$ & BUS NAME & $\begin{array}{c}\text { NO } \\
\text { FACTs }\end{array}$ & $\begin{array}{c}\text { WITH } \\
\text { TCSC }\end{array}$ & $\begin{array}{c}\text { WITH } \\
\text { SSSC }\end{array}$ \\
\hline 47 & Alaoji GS & 1.0 & 1.0 & 1.0 \\
48 & Afam GS & 1.0 & 1.0 & 1.0 \\
\hline Real Power Generated & 57.9435 & 57.8373 & 57.8476 \\
Reactive power & 43.2527 & 42.7683 & 42.9169 \\
$\quad$ Generated & & & \\
Real Power Load & 57.35 & 57.35 & 57.35 \\
Reactive Power Load & 39.52 & 39.52 & 39.52 \\
Real Power Losses & 0.5935 & 0.4873 & 0.4976 \\
Reactive Power Losses & 3.7327 & 3.2483 & 3.3969 \\
$\%$ Decrease of Active & & $17.884 \%$ & $16.15 \%$ \\
Power Losses & & & \\
$\%$ Decrease of Reactive & & $12.979 \%$ & $\mathbf{8 . 9 9 6 \%}$ \\
Power Losses & & & \\
\hline
\end{tabular}

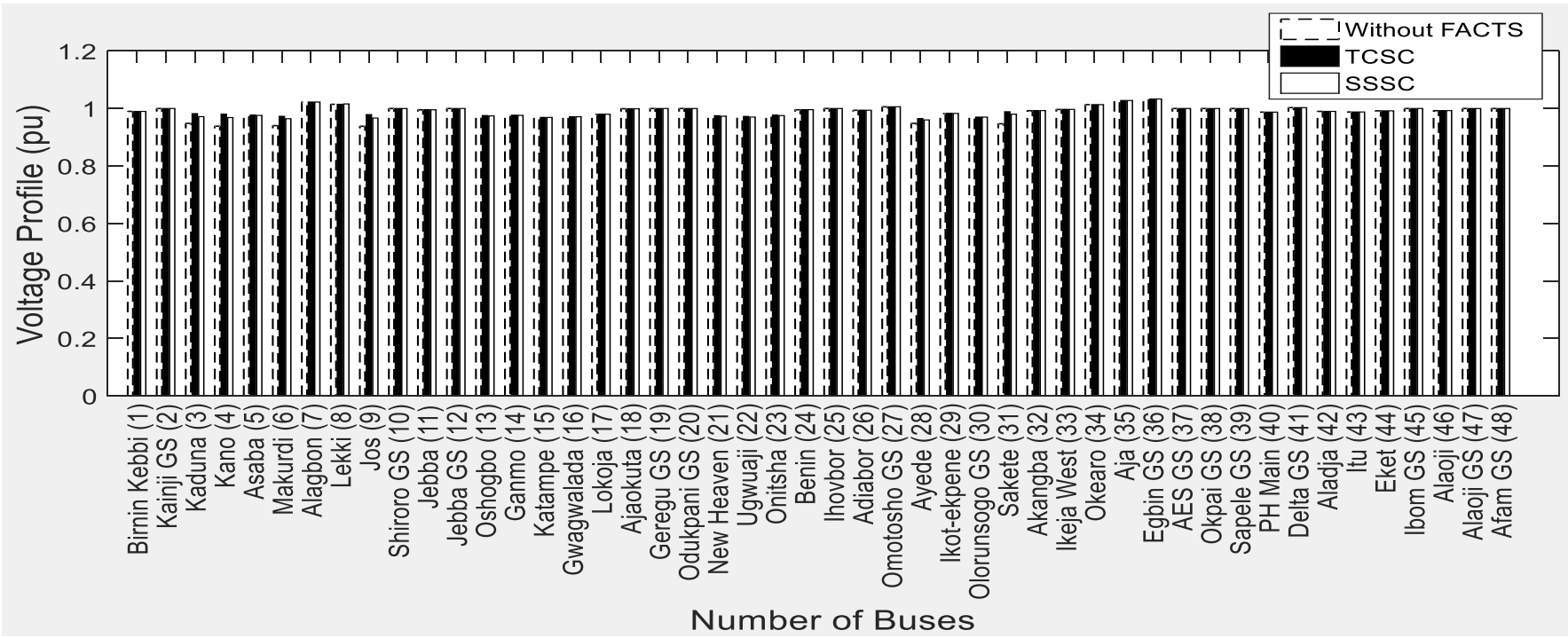

Figure 5: Voltage magnitude profile without and with TCSC and SSSC

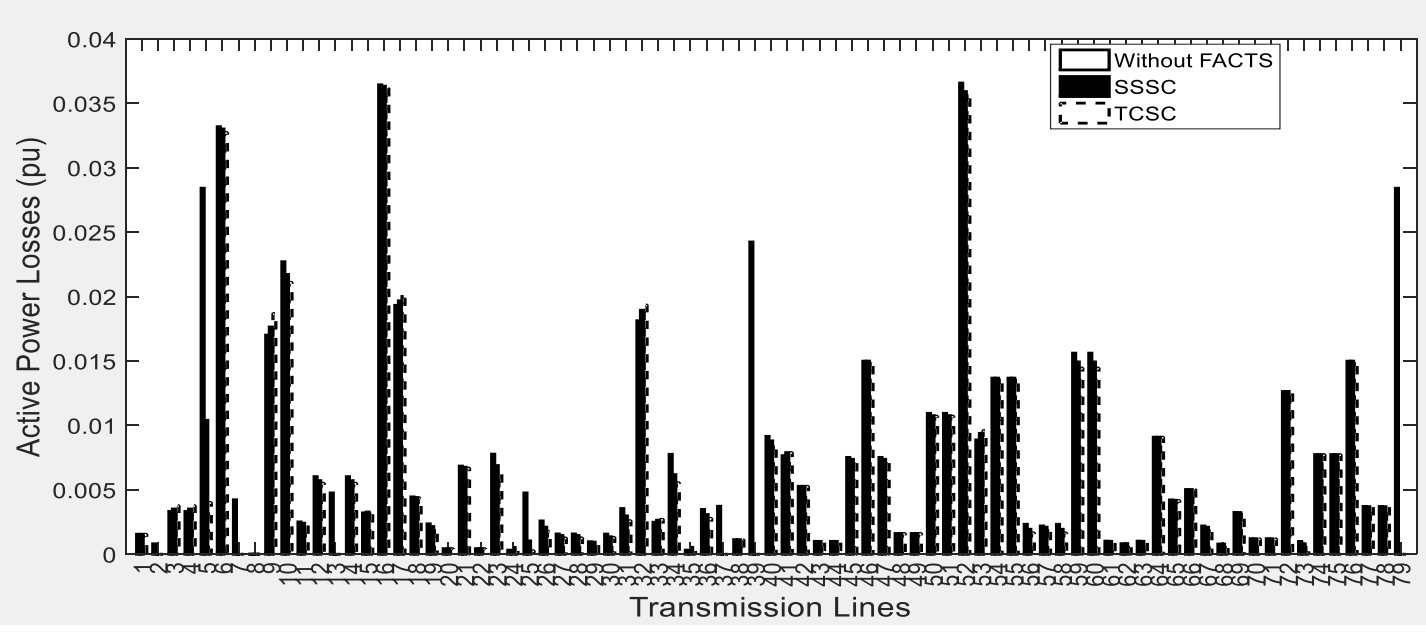

Figure 6(a): Active power losses without and with TCSC and SSSC 


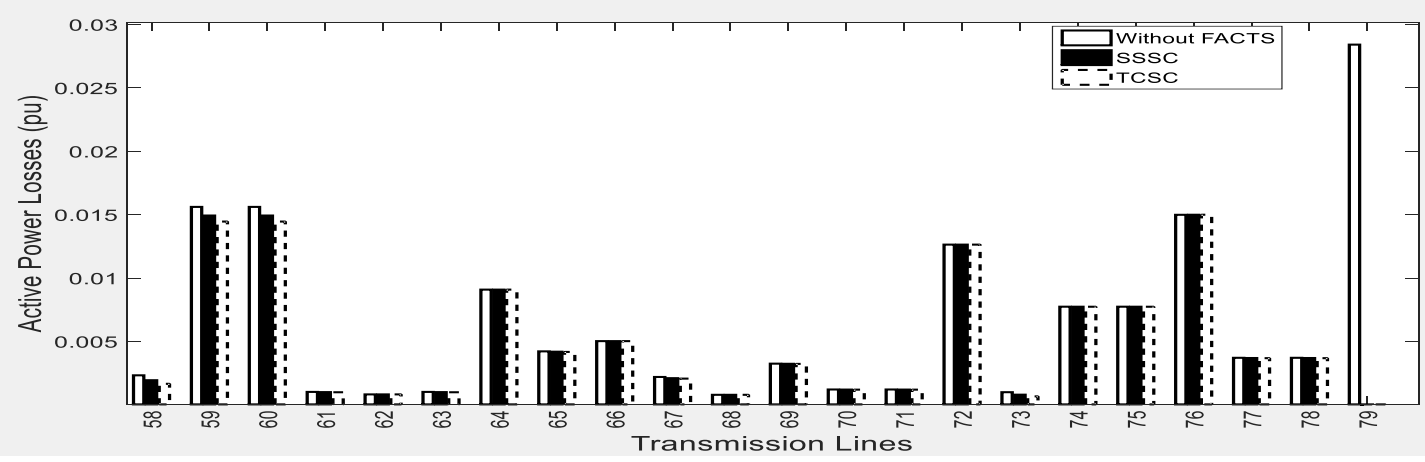

Figure 6(b): A section of Active power losses without and with TCSC and SSSC

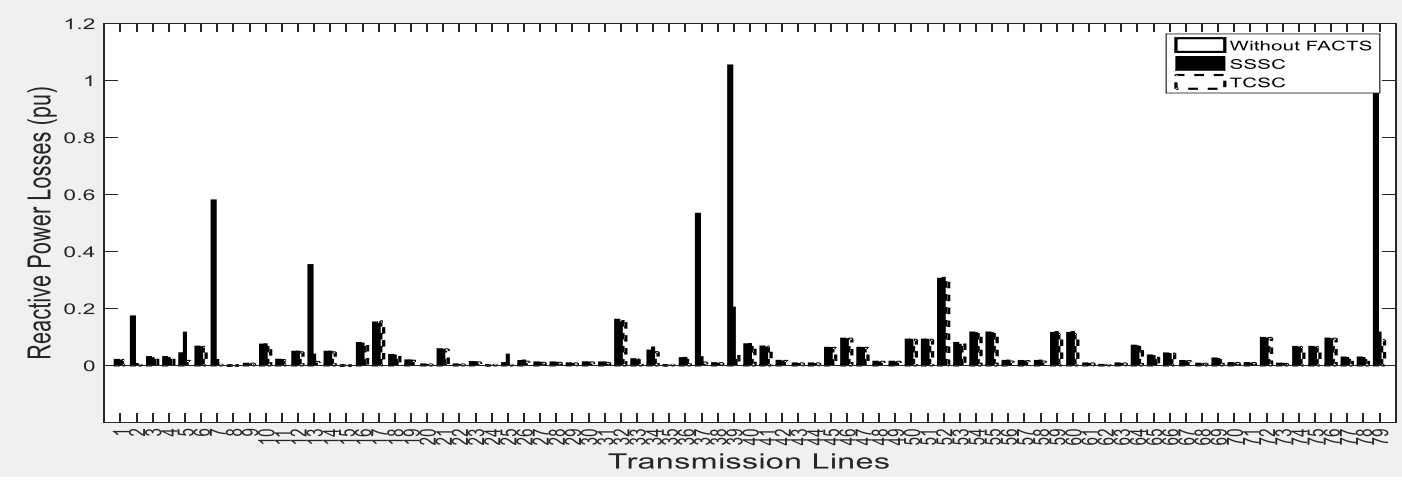

Figure 7 (a): Reactive power losses without and with TCSC and SSSC

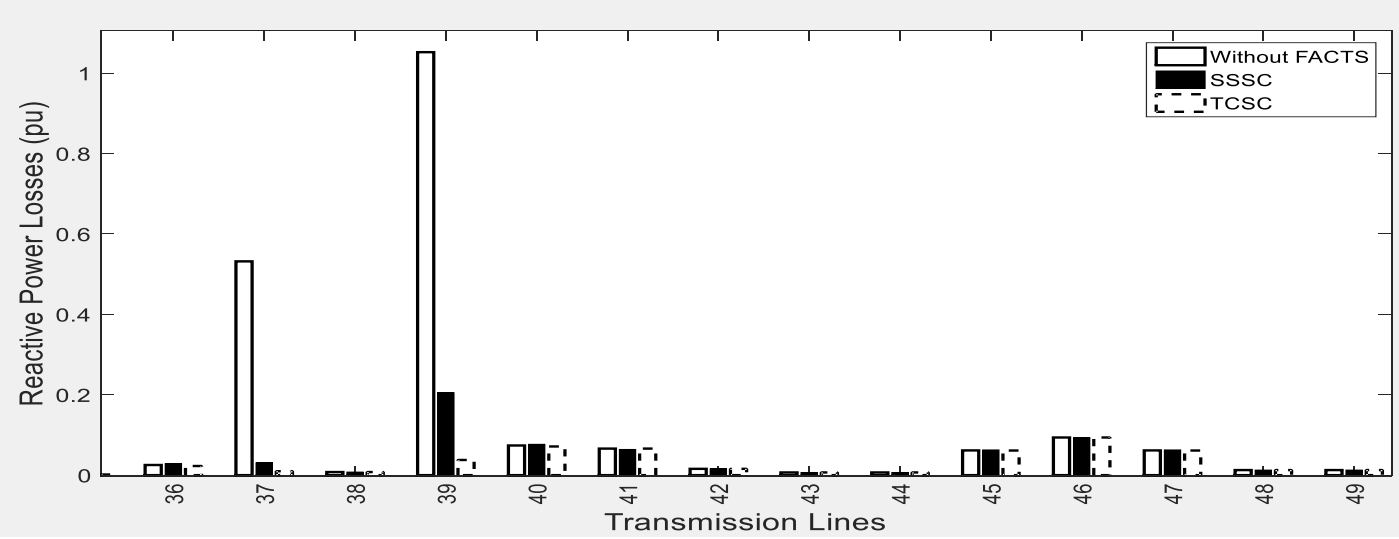

Figure 7(b): A section of the reactive power losses without and with TCSC and SSSC

\section{DISCUSSION OF RESULTS}

The results of the power flow simulation of the network of Figure 3 without FACTS using Newton Raphson iteration method for power flow computations are as presented in Table 3. The iteration is completed in 0.156 second after 4 iterations with a maximum convergence error of 2.9437 e-09 p.u. and active and reactive maximum power mismatches are $2.12 \mathrm{e}-13$ p.u. and $4.01 \mathrm{e}-13$ p.u. respectively. The voltage profile for the unfortified system of Figure 3 and as depicted in Table 3 shows that the following buses have voltage magnitudes below the acceptable $\pm 5 \%$ of the normal $330 \mathrm{kV}$ voltage magnitude profile (1.0 p.u) [19]: 3(Kaduna), 4(Kano), 6(Makurdi), 9(Jos), 28(Ayede) and 31(Sakete). The result of the power flow also showed that the total real power generation in p.u. stands at 57.94345 p.u. and the reactive power at 43.25271 p.u. The total real power load of the system is 57.35 p.u. and the reactive power of the load is $39.52 \mathrm{p}$.u. It can also be seen that the total real power losses in pu is 0.59345 p.u. while the reactive power losses is 3.73251p.u. 
From the Newton Raphson power flow of the system of Figure3, the best position for the installation of TCSC is on line 3-4 closer to the bus with the highest VSSF which was found out to be bus 4(Kano) with 0.57724 as shown in Table 5. The result of the power flow of the network is summarized in Table 6 with the voltage magnitude profile indicated. Power flow is completed in 0.687 second after 4 iterations with a maximum convergence error of $9.6232 \mathrm{e}-11 \mathrm{p} . \mathrm{u}$. and active and reactive maximum power mismatches of 1.07678 p.u. and 1.06881 p.u. respectively. The voltage levels at the buses with dips are raised up to and within $\pm 5 \%$ of the acceptable value. Total real and reactive power generation for the system with TCSC is 57.94345 p.u. and 42.76825 p.u. respectively, while the losses are reduced to 0.48732 p.u. for active power and 3.24825 p.u. for reactive power.

Furthermore, the network of the Nigerian 48 bus system modeled with the installation of SSSC on line 3-4 closer to bus 4 is shown in Figure 4. The power flow of the network also summarized in Table 6 is completed in 0.156 second after 4 iterations with a maximum convergence error of 1.6667 e-10 p.u. The active and reactive maximum power mismatches are 63.19024 p.u. and 46.42357 p.u. respectively. Table 6 shows the voltage magnitude profile with SSSC indicating the improvement of the buses with voltage dips. The total active power losses reduced from 0.59345 p.u. to 0.49761 p.u. and the total reactive power losses reduced from 3.73271 p.u. to 3.39690 p.u. respectively. Graphical representation of the voltage magnitude profile of the buses is shown in Figure 5.

Figures 6 (a) and (b),7(a) and (b) shows the comparison of the active and reactive powers of the system with and without TCSC and SSSC. Computation of the percentage decrease of the real and reactive power losses reflected the capability of the FACTS devices (TCSC and SSSC) to compensate and improve the steady state stability of the power system. From Table 6, the total active power losses dropped from 0.59345 p.u. without FACTS to 0.48732 p.u. with TCSC giving a percentage decrease of $17.884 \%$ while the reactive power dropped from 3.73271 p.u. to 3.24825 p.u; a percentage of $12.979 \%$. For SSSC, the total active power losses dropped from 0.59345 p.u. without FACTS to 0.49761 p.u. giving a percentage decrease of $16.15 \%$ while the reactive power dropped from 3.73271 p.u. to 3.39690 p.u; a percentage of $8.996 \%$.

\section{CONCLUSION}

The steady state stability of the Nigerian 48-bus system has been thoroughly investigated through the use of TCSC and SSSC. The parameters of the system modeled using PSAT without FACTS devices is compared with the system with TCSC and SSSC in the event of small disturbances like voltage drops as a result of long transmission lines, variation in loads etc. The FACTS devices performed creditably by enhancing stability through restoring the voltages at some buses back to an acceptable value and also mitigating against both real and reactive power losses in the system. Of the two devices, TCSC was observed to give a better compensation for effective steady state stability of the Nigerian 48-Bus system compared to SSSC.

\section{REFERENCES}

[1] Kundur, P., Paserba, J., Anderson, G., Bose, A., Canizares, C., Hatziargyriou, N., Hill, D., Stankovic, A., Cutsem, T. and Vittal, V. (2004). Definition and classification of power system stability, IEEE/CIGRE Joint Task Force on Stability Terms and Definitions IEEE Transactions on Power Systems, 19(2): 1387-1401.

[2] Azimoh, L. C., Folly, K. A. and Chowdhury, S. P. (2009). Mitigations of voltage instability in power systems (paper review), IEEE Electrical Power and Energy Conference: 1-6.

[3] Paserba, J. J. (2003). How FACTS controllers benefit AC transmission systems, IEEE PES Transmission and Distribution Conference and Exposition: 991-998.

[4] Rani, M. And Gupta, A. (2014). Steady state voltage stability enhancement of power system using FACTS devices, 6th IEEE Power India International Conference (PIICON): 1-6.

[5] Sanbasiva, N. R, Amarnath, J. and Purnachandra, V. R. (2014). Effects of FACTS devices on enhancement of voltage stability in deregulated power system, IEEE International Conference on Circuit, Power and Computing Technologies (ICCPCT): 642-650.

[6] Folorunso, O., Osuji, C. C. and Ighodalo, O. S. (2014). Enhancement of power system voltage stability with the aid of reactive/capacitive power switching mechanism: (a case study of Owerri Transmission Company of Nigeria), Journal of Advancement in Engineering and Technology, 2(1):1-6, ISSN: 2348-2931.

[7] Aborisade, D. O., Adebayo, I. G. and Oyesina, K. A. (2014). A comparison of the voltage enhancement and loss reduction capabilities of 
STATCOM and SSSC FACTS controllers, American Journal of Engineering Research (AJER), 3(1): 96105.

[8] Shishir, D., Ganga, A., Laxmi, S., and Ankite, S. (2014). An overview of placement of TCSC for enhancement of power system stability, IEEE Sixth International Conference on Computational Intelligence and Communication Networks. 11841187.

[9] Bindeshwar, S., Sharma, N. K., Tiwari, A. N., Verma, K. S., and Deependra, S. (2011). Enhancement of voltage stability by coordinated control of multiple FACTS controllers in multimachine power system environments, IET International Conference on Sustainable Energy and Intelligent Systems: $18-25$.

[10] Ogbuefi, U. C. and Madueme, I. C. (2015). A power flow analysis of the Nigerian $330 \mathrm{kV}$ electric power system, IOSR Journal of Electrical and Electronics Engineering (IOSR-JEEE), 10(1): 4657.

[11] Aribi, F. and Nwohu, M. N. (2014). Optimal location of STATCOM in Nigerian $330 \mathrm{kV}$ network using ant colony optimization meta-heuristic, Global Journal of Research in Engineering: $F$. Electrical and Electronic Engineering, 14(3): 4550.

[12] Adeniji, O. A. and Mbamaluikem, P. O. (2017). Voltage stability enhancement and efficiency improvement of Nigerian transmission system using unified power flow controller, American Journal of Engineering Research (AJER), 6(1): 3743.
[13] Adebayo, I. G., Aborisade, D. O. and Oyesina, K. A. (2013). Steady state voltage stability enhancement using static synchronous series compensator (SSSC); A case study of Nigerian 330 $\mathrm{kV}$ grid system, Research Journal in Engineering and Applied Sciences, 2(1): 54-61.

[14] Hingorani, N. G. and Gyugyi, L. (2000). Understanding FACTS, Concepts \&Technology of Flexible AC Transmission Systems. New York: IEEE.

[15] Kumar, S. A., Easwarlal, C. and Kumar, S. M. (2012). Multi machine power system stability enhancement using static synchronous series compensator (SSSC), IEEE International Conference on Computing, Electronics and Electrical Technologies (ICCEET): 212-217.

[16] Faridi, M., Maeiiat, H., Karimi, M., Farhadiand, P., and Mosleh, H. (2011). Power system stability enhancement using static synchronous series compensator (SSSC), IEEE 3rd International Conference on Computer Research and Development. 387-391.

[17] Zhang, X., Rehtanz, C. and Pal, B. (2012). Flexible AC Transmission System: Modeling and Control, New York: Springer Heidelberg.

[18] PHCN - Transmission Company of Nigeria, National Control Centre, Oshogbo, 2018.

[19] Ayodele, T. R., Ogunjuyigbe, A. S. O. and Oladele, O. O. (2016). Improving the transient stability of Nigerian $330 \mathrm{kV}$ transmission network using static var compensation part 1: the base study, Nigerian Journal of Technology (NIJOTECH), 35(1): 155166. 